\title{
Density Functional Theory Investigation of Substituent Effects on Building Blocks of
}

\author{
Conducting Polymers
}

\author{
U. Salzner \\ Department of Chemistry, Bilkent University, 06533 Bilkent, Ankara, Turkey, e-mail: salzner@fen.bilkent.edu.tr
}

\begin{abstract}
Substituted heterocyclic dimers were calculated employing density functional theory (DFT) and analyzed with the natural bond orbital method (NBO). Substitution in 3- and 4-positions leads to parallel shifting of HOMO and LUMO but does not reduce energy gaps. For bridged dimers, HOMO-LUMO gaps correlate with $\pi$-electron densities in the carbon backbone and energy gap reductions correlate with the strength of $\pi-\pi *$ interactions from the backbone to the bridging group. Alternating donor-acceptor groups do not reduce energy gaps and lead to systems with average HOMO and LUMO levels compared to the parent molecules.
\end{abstract}

Keywords: Density functional calculations, polythiophene and derivatives, polypyrrole and derivatives, low-bandgap conjugated polymers, special-purpose functionalized conjugated polymers, quantum wells

\section{Introduction}

Although various ways have been found to decrease bandgaps of conjugated organic polymers, a true synthetic metal has yet to be designed. The bandgap of polythiophene which is about $2.0 \mathrm{eV}[1]$ has been reduced to $0.8 \mathrm{eV}$ by bridging dimer building blocks with dicyanomethylene groups[2] and to $1.0 \mathrm{eV}$ by grafting benzene rings onto the backbonc.[2] Substitution in 3- and 4-positions has been employed to increase solubility and to create quantum wells but clear trends in bandgaps were not derived.[2]

In this investigation we theoretically examine electronic effects of various substituents in different positions of heteroaromatic dimers (compounds 1-24) and establish correlations which allow to understand and predict substituent effects on bandgaps.

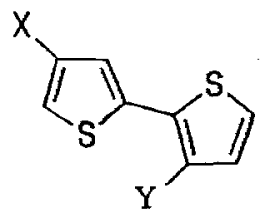

1: $\mathrm{X}=\mathrm{CH}_{3}, \mathrm{Y}=\mathrm{CH}_{3}$

2: $\mathrm{X}=\mathrm{OH}, \mathrm{Y}=\mathrm{OH}$

3: $\mathrm{X}=\mathrm{NH}_{2}, \mathrm{Y}=\mathrm{NH}_{2}$

4: $\mathrm{X}=\mathrm{CN}, \mathrm{Y}=\mathrm{CN}$

5: $\mathrm{X}=\mathrm{CCH}, \mathrm{Y}=\mathrm{CCH}$

6: $\mathrm{X}=\mathrm{OH}, \mathrm{Y}=\mathrm{CN}$

\section{Methods}

Compounds 1 through 24 were optimized using density functional theory (DFT). Becke's three parameter hybrid functional[3] was combined with Perdew and Wang's correlation functional.[3] Stevens Basch Krauss pseudo-potentials[3] and split valence plus polarization basis sets were employed. All molecules were optimized in planar geometry. The use of DFT orbital energies for estimating ionization potentials, electron affinities, and energy gaps is controversial. However, we have shown empirically[4] that hybrid functionals greatly improve the performance of DFT with respect to energy gaps.
With $30 \%$ Hartree-Fock exchange, hybrid functionals yield HOMO-LUMO gaps in close agreement with $\lambda_{\text {max }}$ values from UV spectroscopy. This functional is designated as B3P86-30\% and used throughout. IPs and EAs were shown to be about $1 \mathrm{eV}$ too low for oligomeric $\pi$-systems but trends are reproduced correctly. Substituent effects were analyzed in terms of localized orbitals with the NBO method.[5] All calculations were performed with Gaussian 94.

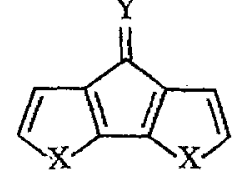

$$
\begin{aligned}
& \text { 7: } X=S, Y=C(C N)_{2} \\
& \text { 8: } X=S, Y=O \\
& \text { 9: } X=S, Y=S \\
& \text { 10: } X=S, Y=C H_{2} \\
& \text { 11: } X=S, Y=C F_{2} \\
& \text { 12: } X=S, Y=C(S C H)_{2} \\
& \text { 13: } X=C H_{2}, Y=C(C N)_{2} \\
& \text { 14: } X=\mathrm{CH}_{2}, Y=O \\
& \text { 15: } X=C_{2}, Y=S \\
& \text { 16: } X=N H Y=C(C N)_{2} \\
& \text { 17: } X=N H Y=O \\
& \text { 18: } X=N H Y=S \\
& \text { 19: } X=O, Y=C(C N)_{2} \\
& \text { 20: } X=O, Y=O \\
& \text { 21: } X=O Y=S \\
& \text { 22: } X=B H Y=C(C N)_{2} \\
& \text { 23: } X=B H Y=O \\
& \text { 24: } X=B H Y=S
\end{aligned}
$$

\section{Results and Discussion}

Table 1 shows that there is very little effect on energy gaps upon 3,4'-substitution (dimers 1-6). Note that there is no gap reduction for donor-acceptor substituted 6 . Comparison of HOMO and LUMO energies reveals that this is due to the almost parallel shift of these energy levels. 
Table 1. Energy gaps and Energy gap reductions compared to the corresponding unsubstituted dimers for 1-24.

\begin{tabular}{cccccccccccccc}
\hline & $\mathbf{1}$ & $\mathbf{2}$ & $\mathbf{3}$ & $\mathbf{4}$ & $\mathbf{5}$ & $\mathbf{6}$ & $\mathbf{7}$ & $\mathbf{8}$ & $\mathbf{9}$ & $\mathbf{1 0}$ & $\mathbf{1 1}$ & $\mathbf{1 2}$ \\
\hline E, & 4.84 & 4.58 & 4.56 & 4.64 & 4.52 & 4.79 & 3.23 & 3.86 & 3.19 & 4.16 & 4.31 & 4.01 \\
E $_{\text {rratik. }}$ & -0.03 & 0.23 & 0.25 & 0.17 & 0.29 & 0.02 & 1.48 & 0.94 & 1.61 & 0.64 & 0.49 & 0.79 \\
HOMO & -6.41 & -6.21 & -5.96 & -7.66 & -6.77 & -6.88 & -7.21 & -6.88 & -6.83 & -6.40 & -6.56 & -6.21 \\
LUMO & -1.58 & -1.63 & -1.40 & -3.02 & -2.24 & -2.09 & -3.98 & -3.02 & -3.64 & -2.25 & -2.24 & -2.20 \\
\hline & $\mathbf{1 3}$ & $\mathbf{1 4}$ & $\mathbf{1 5}$ & $\mathbf{1 6}$ & $\mathbf{1 7}$ & $\mathbf{1 8}$ & $\mathbf{1 9}$ & $\mathbf{2 0}$ & $\mathbf{2 1}$ & $\mathbf{2 2}$ & $\mathbf{2 3}$ & $\mathbf{2 4}$ \\
\hline E, & 2.48 & $\mathbf{3 . 1 5}$ & 2.43 & 3.29 & 4.06 & 3.24 & 3.17 & 3.90 & $\mathbf{3}$ & 10 & 1.70 & 2.11 & 1.21 \\
E $_{\text {rratike }}$ & 1.76 & 1.09 & 1.81 & 2.20 & 1.43 & 2.25 & 2.09 & 1.36 & 2.16 & 1.08 & 0.67 & 1.57 \\
HOMO & -6.48 & -6.18 & -6.09 & -6.46 & -6.02 & -6.02 & -7.11 & -6.76 & -6.69 & -6.76 & -6.56 & -6.23 \\
LUMO & -4.00 & -3.03 & -3.66 & -3.16 & -1.95 & -2.77 & -3.93 & -2.86 & -3.59 & -5.06 & -4.45 & -5.03 \\
\hline
\end{tabular}

HOMO and LUMO energies of 6 are about average between those of 2 and 4 . This also holds for localized $\pi$-NBOs. For 7 24 substantial energy gap reductions result from stronger LUMO energy compared to HOMO energy lowerings.

Substituent effects are strongest on dipyrrole (16-18) and on difuran (19-21) Much smaller effects are found with dicyclopentadiene, dithiophene, and diborole. It is also noteworthy that $\mathrm{C}=\mathrm{S}$ leads to slightly stronger energy gap reductions than $\mathrm{C}=\mathrm{C}(\mathrm{CN})_{2}$. The bandgap reduction of poly -7 has been discussed in terms of alternating donor-acceptor substitution.[6] According to this argument, dipyrrole 16 should have an even smaller energy gap than 7 since nitrogen is a stronger donor than sulfur. This is, however, not the case. The lowest energy gap is found for diborole 24 which contains two acceptors, BH and $\mathrm{C}=\mathrm{S}$.

These trends can be rationalized with $\mathrm{NBO}$ analysis. Figs. 1 and 2 show that energy gaps correlate with $\pi$-electron densities in the carbon backbone and that energy gap reductions compared to parent dimers correlate with the strength of the $\mathrm{C}=\mathrm{C}->\mathrm{C}=\mathrm{Y}^{*}$

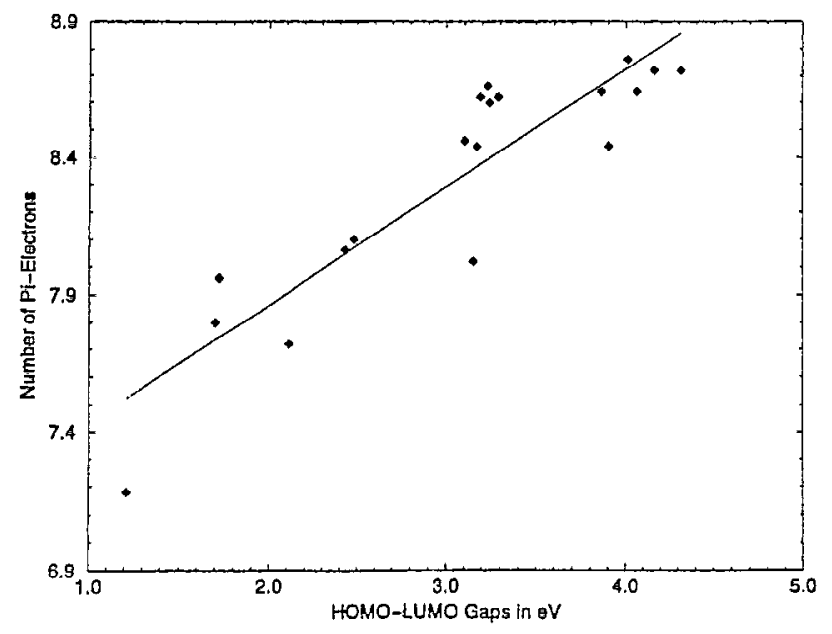

Fig. 1

orbital interactions. No correlation was found between total charges in the carbon backbone and energy gaps. The comparatively small effects of bridging groups on bithiophene

[1] J.-L. Brédas, In T. A. Skotheim, (ed.), Handbook of Conducting Polymers, Dekker: NY, 1986, pp 859-913.

[2] J. Roncali, Chem. Rev. (1997) 97, 173.

[3] M. J. Frisch, Æ. Frisch, J. B. Foresman, Gaussian 94 User's Reference, Gaussian, Inc.: Pittsburgh, 1994-1995.

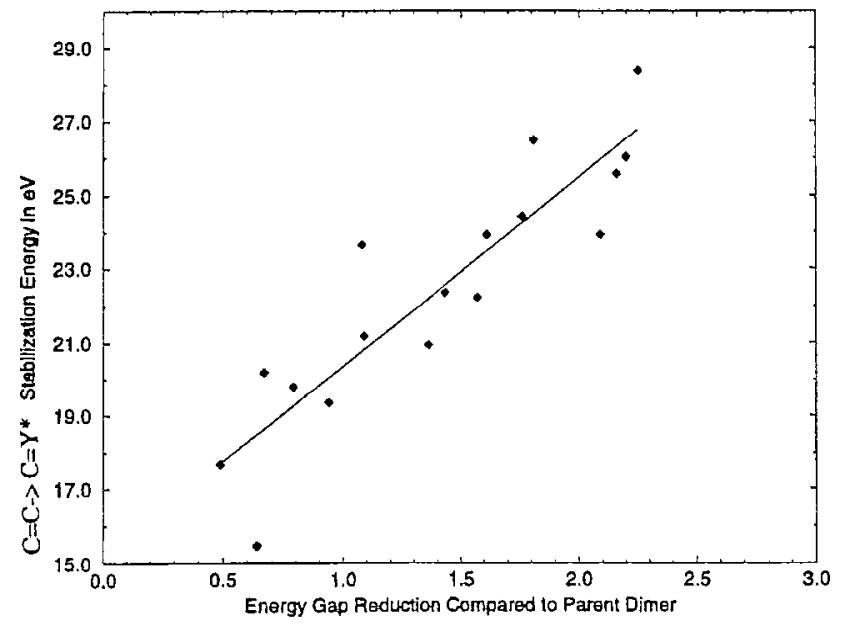

Fig. 2

can be traced back to the ability of the sulfur lone pairs to compensate for electron withdrawing effects of substituents. While occupations of the sulfur lone pairs differ by up to $0.05 \mathrm{e}$ in 1-12, occupations of $N$ lone pairs in 16-18 are constant and identical to that in dipyrrole. Therefore dipyrrole shows the largest substituent effects of all heteroaromats studied here. Since energy gaps correlate with $\pi$-electron densities, energy gaps are smallest if two acceptor groups rather than alternating donor-acceptor substituents are present. $\mathrm{C}=\mathrm{S}$ groups are as effective as $\mathrm{C}=\mathrm{C}(\mathrm{CN})_{2}$ since the $\mathrm{C}=\mathrm{S}^{*}$ orbital lies very low and is an excellent electron acceptor.

\section{Conclusions}

To decrease energy gaps, $\pi$-electron density needs to be withdrawn from the $\pi$-backbone. To prevent parallel shift of HOMO and LUMO levels, substituents have to be placed in positions where HOMOs have nodes. Donor-acceptor substitution does not reduce energy gaps and does not create quantum well structures in the ground state of conjugated organic polymers. This is due to the delocalized nature of the $\pi$-system which prevents local accumulation of charge.

[4] U. Salzner, J. B. Lagowski, P. G. Pickup, R. A. Poirier, J. Comput. Chem. (1997) 18, 1943.

[5] NBO Version 3.1, E. D. Glending, A. E. Reed, J. E. Carpenter, F. Weinhold.

[6] A. K.Bakhshi, Y. Yamaguchi, H. Ago, T. Yamabe, Synth. Met. (1996) 79, 115. 